\title{
ON THE EXISTENCE OF MEROMORPHIC FUNCTIONS WITH PREASSIGNED ASYMPTOTIC SPOTS
}

\author{
By GenKō HiROMI
}

In his paper [2], Heins introduced the notion of asymptotic spot of an interior transformation and then in [3], especially, he examined asymptotic spots of entire and meromorphic functions. Let $f(z)$ be meromorphic in $|z|<\infty$, and let $w_{0}$ denote a point of the extended $w$-plane. Then $\sigma$ is called an asymptotic spot over $w_{0}$ when $\sigma$ is a function (a correspondence from sets to sets) whose domain is the family $\Phi_{w_{0}}$ of simply-connected Jordan regions containing $w_{0}$ and which satisfies: (a) for each $\Omega \in \Phi_{w_{0}}, \sigma(\Omega)$ is a component of $f^{-1}(\Omega)$ which is not relatively compact, and (b) if $\Omega_{1} \subset \Omega_{2}$, for $\Omega_{1}, \Omega_{2} \in \Phi_{w_{0}}$, then $\sigma\left(\Omega_{1}\right) \subset \sigma\left(\Omega_{2}\right)$. Let $\mathbb{S}_{\Omega}\left(w, w_{0}\right)$ denote Green's function for $\Omega$ with the pole at $w_{0}$. We put

$$
u_{\sigma(\Omega)}(z) \equiv \text { G.H.M. } \mathbb{S}_{\Omega}\left(f_{\sigma(\Omega)}(z), w_{0}\right),
$$

where $f_{\sigma(\Omega)}(z)$ is the restriction of $f(z)$ to $\sigma(\Omega)$ and G.H.M. means the greatest hermonic minorant. We associate with the pair $(\sigma, \Omega)$ an index $h(\sigma, \Omega)$ as follows. If $u_{\sigma(\Omega)}(z) \equiv 0$, then $h(\sigma, \Omega)=0$. If $u_{\sigma(\Omega)}(z)>0$ and is represented as a finite sum of $n$ mutually non-proportional minimal positive harmonic functions on $\sigma(\Omega)$, then $h(\sigma, \Omega)=n$. In the remaining case, we set $h(\sigma, \Omega)=+\infty$. The index $h(\sigma, \Omega)$ is monotone in $\Omega$, i.e. if $\Omega_{1} \subset \Omega_{2}$, then $h\left(\sigma, \Omega_{1}\right) \leqq h\left(\sigma, \Omega_{2}\right)$. The harminic index $h(\sigma)$ of $\sigma$ is then defined as

$$
\inf _{\Omega \in \Phi_{w_{0}}} h(\sigma, \Omega)
$$

Now Heins proposed the following realization problem: Let $w_{1}, \cdots, w_{n}$ denote $n(\geqq 1)$ given points on the extended $w$-plane and $h_{1}, \cdots, h_{n}$ denote $n$ given positive integers. Does there exist a meromorphic function $f(z)$ in $|z|<\infty$ which satisfies: (I) the asymptotic spots of $f(z)$ having positive harmonic indices are $n$ in number, say $\sigma_{1}, \cdots, \sigma_{n}$, (II) $\sigma_{k}$ lies over $w_{k}$ and $h\left(\sigma_{k}\right)=h_{k}$, (III) $f(z)$ is of order $H / 2$, where $H=\sum_{k=1}^{n} h_{k}$ ?

The object of the present paper is to give a solution for this problem.

Heins showed an affirmative answer for the special cases: (i) $n=1$, (ii) $n=2$, $h_{1}=h_{2}=2$. As a direct consequence of the method which Heins used to construct an example of the case (ii), M. Ozawa has informed to the author an affirmative answer for the case (iii) $n=2, h_{1}=h_{2}=m$. In fact, it is shown that the argument similar to the case (ii) in [3] (p. 439) remains valid in the case (iii) by considering the starting function $g(z)=e^{-\imath z} \cos z^{m}$ in place of $g(z)=e^{-\imath z} \cos z^{2}$.

Received December 10, 1964. 
Here we shall give an answer for the case: $n=2, h_{1}, h_{2}, w_{1}$ and $w_{2}$ unrestricted and further solve the general problem affimatively.

To this end we need some preparatory considerations. Suppose that $G$ is a Jordan region in $|z|<\infty$ and that $U$ is a harmonic function non-negative on $G$ which vanishes on the boundary of $G$. Further suppose that $\left\{G_{k}\right\}$ is a family of Jordan subregions of $G$ satisfying $G_{k} \cap G_{l}=\phi$ for $k \neq l$, and that $U_{k}$ is a harmonic function non-negative on $G_{k}$ which vanishes continuously on the boundary of $G_{k}$ and is dominated by $U$ on $G_{k}$. Let $U_{k}^{*}$ denote the least harmonic majorant of the subharmonic function which is equal to $U_{k}$ on $G_{k}$ and to zero on $G-G_{k}$. Then we get the following lemma.

LEMMA. Under the above assumption it holds

$$
\Sigma U_{k}^{*} \leqq U
$$

if each $U_{k}$ is minimal in $G_{k}$, then $U_{1}^{*}, U_{2}^{*}, \cdots$ are minimal and mutually non-proportional in $G$.

The proof of the lemma is contained in (f) and (c) of [2] (pp. 442-445).

In [3], Heins formulated the Denjoy-Carleman-Ahlfors theorem and gave the following theorem (p. 431).

Theorem A. Let $H$ denote the grand total of the harmonic indices of all the asymptotic spots of a non-constant meromorphic function $f$ in $|z|<\infty$. Let $T(r ; f)$ denote the Nevanlinna characteristic function of $f$. If $H=+\infty$, then

$$
\lim _{r \rightarrow \infty} \frac{\log T(r ; f)}{\log r}=+\infty
$$

If $2 \leqq H<\infty$, then

$$
\lim _{r \rightarrow \infty} \inf \frac{T(r ; f)}{r^{H / 2}}>0 .
$$

If $H=1$ and the asymptotic spot $\sigma_{0}$ with index one is such that for some $\Omega$ of its domain, the complement of $\sigma_{0}(\Omega)$ intersects all circles $\{|z|=r\}$ with $r$ sufficiently large, then

$$
\lim _{r \rightarrow \infty} \frac{T(r ; f)}{r^{1 / 2}}>0 .
$$

Now we observe Mittag-Leffler's function

$$
E_{\alpha}(z)=\sum_{n=0}^{\infty} \frac{z^{n}}{\Gamma(1+\alpha n)} \quad(0<\alpha<2)
$$

which is an entire function of order $1 / \alpha$ and quote the following theorem (cf. $\S 3.62$ in [1]):

If $0<\alpha<1$ there exists a constant $K$ independent of $\alpha$ such that

$$
\left|E_{\alpha}(z)-\frac{\exp z^{1 / \alpha}}{\alpha}+\frac{1}{z \Gamma(1-\alpha)}\right| \leqq \frac{K}{\alpha^{2}|z|^{2}} \text { for }|\arg z| \leqq \frac{3}{4} \alpha \pi,|z| \geqq 2,
$$


(2) $\left|E_{\alpha}(z)+\frac{1}{z \Gamma(1-\alpha)}\right| \leqq \frac{K}{\alpha^{2}|z|^{2}} \quad$ for $\quad \frac{3}{4} \alpha \pi \leqq \arg z \leqq 2 \pi-\frac{3}{4} \alpha \pi,|z| \geqq 2$.

By using Mittag-Leffler's function $E_{\alpha}(z)$, we put

$$
f_{k}(z)=E_{2 / \boldsymbol{H}}\left(z \varepsilon^{k-1}\right) \quad(k=1,2, \cdots, H(\geqq 3)),
$$

where $\varepsilon$ is a primitive $H$-th root of $1: \varepsilon=\cos (2 \pi / H)-i \sin (2 \pi / H)$.

Proposition 1. The function

$$
f(z)=\sum_{j=1}^{h_{1}} f_{j}(z) / \sum_{\jmath=h_{1}+1}^{H} f_{j}(z)
$$

has the desired properties for the case: $n=2, w_{1}=\infty, w_{2}=0$ and $H \geqq 3$.

Proof. We define an asymptotic spot $\sigma_{1}$ over $w_{1}=\infty$ as follows. For $-\pi / H$ $\leqq \arg z \leqq-\pi / 2 H$

$$
\begin{aligned}
&\left|f_{1}(z)\right| \geqq \frac{H}{2}\left|\exp z^{H / 2}\right|-\frac{1}{|z| \Gamma(1-2 / H)}-\frac{H^{2} K}{4|z|^{2}} \\
&\left|f_{H}(z)\right| \leqq \frac{H}{2}\left|\exp z^{-H / 2}\right|+\frac{1}{|z| \Gamma(1-2 / H)}-\frac{H^{2} K}{4|z|^{2}} \leqq \frac{H}{2}+\frac{1}{|z| \Gamma(1-2 / H)}+\frac{H^{2} K}{4|z|^{2}}, \\
&\left|f_{j}(z)\right| \leqq \frac{1}{|z| \Gamma(1-2 / H)}+\frac{H^{2} K}{4|z|^{2}} \quad(j=2, \cdots, H-1) ; \\
&|f(z)| \geqq\left\{\left|f_{1}(z)\right|-\sum_{\jmath=2}^{h_{1}}\left|f_{j}(z)\right|\right\} / \sum_{\jmath=h_{1}+1}^{H}\left|f_{j}(z)\right| \\
& \geqq\left\{\left|\exp z^{H / 2}\right|-\frac{2}{|z| \Gamma(1-2 / H)}-\frac{H^{2} K}{2|z|^{2}}\right\} /\left\{1+\frac{2}{|z| \Gamma(1-2 / H)}+\frac{H^{2} K}{2|z|^{2}}\right\} .
\end{aligned}
$$

For $|\arg z-2(k-1) \pi / H| \leqq \pi / 2 H\left(k=1, \cdots, h_{1}\right)$

$$
\begin{aligned}
\left|z f_{k}(z)\right| & \geqq \frac{H}{2}\left|\exp \left(z \varepsilon^{k-1}\right)^{H / 2}\right|-\frac{1}{\Gamma(1-2 / H)}-\frac{H^{2} K}{4|z|}, \\
\left|z f_{j}(z)\right| & \leqq \frac{1}{\Gamma(1-2 / H)}+\frac{H^{2} K}{4|z|} \quad(j=1, \cdots, k-1, k+1, \cdots, H) \\
|f(z)| & \geqq\left\{\left|z f_{k}(z)\right|-\sum_{j=1}^{k-1}\left|z f_{j}(z)\right|-\sum_{\jmath=k+1}^{h_{1}}\left|z f_{j}(z)\right|\right\} / \sum_{\jmath=h_{1}+1}^{H}\left|z f_{j}(z)\right| \\
& \geqq\left\{\left|z \exp \left(z \varepsilon^{k-1}\right)^{H / 2}\right|-\frac{2}{\Gamma(1-2 / H)}-\frac{H^{2} K}{2|z|}\right\} /\left\{\frac{2}{\Gamma(1-2 / H)}+\frac{H^{2} K}{2|z|}\right\} \\
& \geqq\left\{\left|z \cosh \left(z \varepsilon^{k-1}\right)^{H / 2}\right|-\frac{2}{\Gamma(1-2 / H)}-\frac{H^{2} K}{2|z|}\right\} /\left\{\frac{2}{\Gamma(1-2 / H)}+\frac{H^{2} K}{2|z|}\right\} .
\end{aligned}
$$

For $|\arg z+\pi / H-2 k \pi / H| \leqq \pi / 2 H\left(k=1, \cdots, h_{1}-1\right)$

$$
\left|z f_{k}(z)-\frac{H}{2} z \exp \left(z \varepsilon^{k-1}\right)^{H / 2}\right| \leqq \frac{1}{\Gamma(1-2 / H)}+\frac{H^{2} K}{4|z|},
$$




$$
\begin{aligned}
\left|z f_{k+1}(z)-\frac{H}{2} z \exp \left(z \varepsilon^{k}\right)^{H / 2}\right| \leqq \frac{1}{\Gamma(1-2 / H)}+\frac{H^{2} K}{4|z|}, & \\
\left|z f_{j}(z)\right| & \leqq \frac{1}{\Gamma(1-2 / H)}+\frac{H^{2} K}{4|z|} \quad(j=1, \cdots, k-1, k+2, \cdots, H) \\
|f(z)| & \geqq\left\{\left|z f_{k}(z)+z f_{k+1}(z)\right|-\sum_{j=1}^{k-1}\left|z f_{j}(z)\right|-\sum_{\jmath=k+2}^{h_{1}}\left|z f_{j}(z)\right|\right\} / \sum_{\jmath=h_{1}+1}^{H}\left|z f_{j}(z)\right| \\
& \geqq\left\{\left|z \cosh z^{H / 2}\right|-\frac{1}{\Gamma(1-2 / H)}-\frac{H^{2} K}{4|z|}\right\} /\left\{\frac{2}{\Gamma(1-2 / H)}+\frac{H^{2} K}{4|z|}\right\} .
\end{aligned}
$$

And for $\pi / 2 H+2\left(h_{1}-1\right) \pi / H \leqq \arg z \leqq \pi / H+2\left(h_{1}-1\right) \pi / H$

$$
\begin{aligned}
& \left|f_{h_{1}}(z)\right| \geqq \frac{H}{2}\left|\exp \left(z \varepsilon^{h_{1}-1}\right)^{H / 2}\right|-\frac{1}{|z| \Gamma(1-2 / H)}-\frac{H^{2} K}{4|z|^{2}} \\
& \left|f_{h_{1}+1}(z)\right| \leqq \frac{H}{2}\left|\exp \left(z \varepsilon^{h_{1}}\right)^{H / 2}\right|+\frac{1}{|z| \Gamma(1-2 / H)}+\frac{H^{2} K}{4|z|^{2}} \\
& \quad \leqq \frac{H}{2}+\frac{1}{|z| \Gamma(1-2 / H)}+\frac{H^{2} K}{4|z|^{2}}, \\
& \left|f_{j}(z)\right| \leqq \frac{1}{|z| \Gamma(1-2 / H)}+\frac{H^{2} K}{4|z|^{2}} \quad\left(j=1, \cdots, h_{1}-1, h_{1}+2, \cdots, H\right) ; \\
& |f(z)| \geqq\left\{\left|f_{h_{1}}(z)\right|-\sum_{j=1}^{h_{1}-1}\left|f_{j}(z)\right|\right\} / \sum_{j=h_{1}+1}^{H}\left|f_{j}(z)\right| \\
& \geqq\left\{\left|\exp \left(z \varepsilon^{h_{1-1}}\right)^{H / 2}\right|-\frac{2}{|z| \Gamma(1-2 / H)}-\frac{H^{2} K}{2|z|^{2}}\right\} /\left\{1+\frac{2}{|z| \Gamma(1-2 / H)}+\frac{H^{2} K}{2|z|^{2}}\right\} .
\end{aligned}
$$

From these inequalities we see that if $M$ is sufficiently large the open set $\{z ;|f(z)|$ $>M\}$ contains the union $G_{1}$ of regions

$$
\begin{aligned}
& \left\{z ;\left|\exp z^{H / 2}\right|>M^{2},-\frac{\pi}{H}<\arg z \leqq-\frac{\pi}{2 H}\right\} \\
& \left\{z ;\left|z \cosh z^{H / 2}\right|>M^{2},-\frac{\pi}{2 H} \leqq \arg z \leqq \frac{\pi}{2 H}+\frac{2\left(h_{1}-1\right) \pi}{H}\right\}
\end{aligned}
$$

and

$$
\left\{z ;\left|\exp \left(z \varepsilon^{h_{1}-1}\right)^{I I / 2}\right|>M^{2}, \frac{\pi}{2 H}+\frac{2\left(h_{1}-1\right) \pi}{H} \leqq \arg z<\frac{\pi}{H}+\frac{2\left(h_{1}-1\right) \pi}{H}\right\} .
$$

Clearly the set $G_{1}$ is an unbounded region. We define an asymptotic spot $\sigma_{1}$ over $w_{1}=\infty$ by putting $\sigma_{1}(|w|>M) \equiv$ the component of $f^{-1}(|w|>M)$ containing $G_{1}$. Clearly for every $\Omega \in \Phi_{w_{1}}, \sigma(\Omega)$ is well defined suitably. Next we get $h\left(\sigma_{1}\right) \geqq h_{1}$. In fact, for sufficiently large $M$ the inequality

$$
\log \frac{|f(z)|}{M} \geqq U_{k} \equiv \operatorname{Re}\left(z \varepsilon^{k-1}\right)^{H / 2}-2 \log M
$$


holds in the region

$$
\Delta_{k}:\left\{z ; U_{k}(z)>0,-\frac{\pi}{H}+\frac{2(k-1) \pi}{H}<\arg z<\frac{\pi}{H}+\frac{2(k-1) \pi}{H}\right\}, k=1, \cdots, h_{1} .
$$

Now $\log (|f(z)| / M)$ being superharmonic, $u_{\sigma_{1}(|w|>M)}(z) \equiv$ G.H.M. $\log (|f(z)| / M)$ is nonnegative and $u_{\sigma_{1}(|w|>M)}(z) \geqq U_{k}(z)$ in $\Delta_{k}$. Since $U_{k}(z)$ is minimal in $\Delta_{k}, u_{\sigma_{1}(|w|>M)}(z)$ dominates at least $h_{1}$ mutually non-proportional minimal functions by Lemma. Therefore we get $h\left(\sigma_{1},|w|>M\right) \geqq h_{1}$ for every large $M$, and hence $h\left(\sigma_{1}\right) \geqq h_{1}$.

Similarly we can find an asymptotic spot $\sigma_{2}$ over $w_{2}=0$ having $h\left(\sigma_{2}\right) \geqq h_{2}$. In fact, let a set $G_{2}$ be the union of regions

$$
\begin{aligned}
& \left\{z ;\left|\exp \left(z \varepsilon^{h_{1-1}}\right)^{H / 2}\right|>M^{2},-\frac{\pi}{H}+\frac{2 h_{1} \pi}{H}<\arg z \leqq-\frac{\pi}{2 H}+\frac{2 h_{1} \pi}{H}\right\}, \\
& \left\{z ;\left|z \cosh z^{H / 2}\right|>M^{2},-\frac{\pi}{2 H}+\frac{2 h_{1} \pi}{H} \leqq \arg z \leqq \frac{\pi}{2 H}+\frac{2(H-1) \pi}{H}\right\}
\end{aligned}
$$

and

$$
\left\{z ;\left|\exp \left(z \varepsilon^{H-1}\right)^{H / 2}\right|>M^{2}, \frac{\pi}{2 H}+\frac{2(H-1) \pi}{H}-\leqq \arg z<\frac{\pi}{H}+\frac{2(H-1) \pi}{H}\right\} .
$$

Then the set $\{z ;|f(z)|<1 / M\}$ contains $G_{2}$. If an asymptotic spot $\sigma_{2}$ over $w_{2}=0$ is defined by putting $\sigma_{2}(|w|<1 / M) \equiv$ the component of $f^{-1}(|w|<1 / M)$ containing $G_{2}$, we get $h\left(\sigma_{2}\right) \geqq h_{2}$ by the argument similar to the case of $\sigma_{1}$.

The order $\rho$ of $f(z)$ is at most $H / 2$ since $E_{\alpha}(z)$ is of order $1 / \alpha$. On the other hand, we get, by Theorem $\mathrm{A}, \bar{H} \leqq 2 \rho$ for the grand total $\bar{H}$ of the harmonic indices of all the asymptotic spots of $f$. Consequently we have

and hence

$$
H=h_{1}+h_{2} \leqq h\left(\sigma_{1}\right)+h\left(\sigma_{2}\right) \leqq \bar{H} \leqq 2 \rho \leqq H,
$$

$$
\rho=\frac{H}{2}, h\left(\sigma_{1}\right)=h_{1}, h\left(\sigma_{2}\right)=h_{2} \text { and } \bar{H}=H .
$$

We thus obtain the desired result.

For arbitrary $w_{1}$ and $w_{2}$, if $w_{1} \neq w_{2}$ it suffices to consider a function $L \circ f$ where $L$ is a linear fractional transformation satisfying $L(\infty)=w_{1}, L(0)=w_{2}$, and if $w_{1}=w_{2}$ it suffices to consider a function $f+1 / f$ or $1 /(f+1 / f)+w_{1}$ according to $w_{1}=\infty$ or $w_{1} \neq \infty$. Here we remark that a set $\{z ;|f+1 / f|>M\}$ has two desired unbounded components. For on the half rays $\{z ; \arg z=-\pi / H\}$ and $\left\{z ; \arg z=\pi / H+2\left(h_{1}-1\right) \pi / H\right\}$ we get $|f+1 / f| \leqq 3$ for every large $|z|$.

The assumption $H \geqq 3$ is not essential. For if $H=2$ and $n=2$ we have a particular function $\exp z$ as the above $f$.

Now we shall treat the general problem. Let $w_{1}, \cdots, w_{n}(n \geqq 3)$ denote $n$ given points in the extended $w$-plane, and $h_{1}, \cdots, h_{n}$ denotes $n$ given positive integers. We suppose without loss of generality that the set $\left\{w_{k} ; k=1, \cdots, n\right\}$ does not contain the point at infinity. For the required properties are invariant under any linear fractional transformation of values of an admissible function. 
Again by using Mittag-Leffler's function, we put

$$
f_{j}(z)=E_{2 / H}\left(z \varepsilon^{\jmath-1}\right) \quad\left(j=1, \cdots, H\left(=h_{1}+\cdots+h_{n} \geqq 3\right)\right),
$$

where $\varepsilon$ is a primitive $H$-th root of 1 : $\varepsilon=\cos (2 \pi / H)-i \sin (2 \pi / H)$. From $f_{j}(z)$ we construct a function $\tilde{f}_{k}(z)$ associated with $h_{k}$ as follows. If $h_{k}=1$, we put

If $h_{k}>1$ we put

$$
\tilde{f}_{k}(z)=f_{1}(z) \text {. }
$$

$$
\tilde{f}_{k}(z)=\left(\sum_{j=1}^{h_{k}} f_{j}(z)\right) g_{k}(z)
$$

where $g_{k}(z)$ is defined by

$$
g_{k}(z)= \begin{cases}E_{2 h_{k} / H}\left(z \varepsilon^{\left(h_{k}-1\right) / 2}\right) & \text { for } 2 h_{k}<H, \\ E_{2\left(H-h_{k}\right) / H}\left(z \varepsilon^{H+h_{k}-1}\right)^{-1} & \text { for } 2 h_{k}>H, \\ \exp z \varepsilon^{\left(h_{k}-1\right) / 2} & \text { for } 2 h_{k}=H .\end{cases}
$$

Proposition 2. The function

$$
f(z)=\left\{\sum_{k=1}^{n} w_{k} \tilde{f}_{k}\left(z \varepsilon^{h_{1}+\cdots+h_{k-1}}\right)+A\right\} / \sum_{k=1}^{n} \tilde{f}_{k}\left(z \varepsilon^{h_{1}+\cdots+h_{k-1}}\right)
$$

has the required properties provided $A$ is a sufficiently large number.

Proof. We first examine the properties of $f_{k}(z)$. From the estimations obtained in (1) and (2) we get

$$
\begin{aligned}
& \left|\sum_{j=1}^{h_{k}} f_{j}(z)\right| \geqq \frac{H}{2}\left|\cosh z^{H / 2}\right|-\frac{H}{|z| \Gamma(1-2 / H)}-\frac{H^{3} K}{4|z|^{2}} \\
& \text { for }-\frac{\pi}{H} \leqq \arg z \leqq-\frac{\pi}{H}+\frac{2 h_{k} \pi}{H}, \\
& \left|\sum_{j=1}^{h_{k}} f_{j}(z)\right| \leqq \frac{H}{2}\left|\exp \left(z \varepsilon^{h_{k}-1}\right)^{H / 2}\right|+\frac{H}{|z| \Gamma(1-2 / H)}+\frac{H^{3} K}{4|z|^{2}} \\
& \text { for }-\frac{\pi}{H}+\frac{2 h_{k} \pi}{H} \leqq \arg z \leqq-\frac{\pi}{2 H}+\frac{2 h_{k} \pi}{H}, \\
& \left|\sum_{j=1}^{h_{k}} f_{j}(z)\right| \leqq \frac{H}{|z| \Gamma(1-2 / H)}+\frac{H^{3} K}{4|z|^{2}} \\
& \text { for }-\frac{\pi}{2 H}+\frac{2 h_{k} \pi}{H} \leqq \arg z \leqq 2 \pi-\frac{3 \pi}{2 H}, \\
& \left|\sum_{j=1}^{h_{k}} f_{j}(z)\right| \leqq \frac{H}{2}\left|\exp z^{I I / 2}\right|+\frac{H}{|z| \Gamma(1-2 / H)}+\frac{H^{3} K}{4|z|^{2}} \\
& \text { for }-\frac{3 \pi}{2 H} \leqq \arg z \leqq-\frac{\pi}{H} \text {. }
\end{aligned}
$$

Hence for sufficiently large $|z|$ we have 


$$
\begin{array}{ll}
\left|\sum_{j=1}^{h_{k}} f_{j}(z)\right| \geqq \frac{H}{4}\left|\cosh z^{H / 2}\right| & \text { for }-\frac{\pi}{H} \leqq \arg z \leqq-\frac{\pi}{H}+\frac{2 h_{k} \pi}{H}, \\
\left|\sum_{j=1}^{h_{k}} f_{j}(z)\right| \leqq H & \text { for }-\frac{\pi}{H}+\frac{2 h_{k} \pi}{H} \leqq \arg z \leqq 2 \pi-\frac{\pi}{H} .
\end{array}
$$

Concerning $g_{k}(z)$ we have

$$
\begin{gathered}
\left|g_{k}(z)\right| \geqq \frac{H}{2 h_{k}}\left|\exp \left(z \varepsilon^{\left(h_{k}-1\right) / 2}\right)^{H / 2 h_{k}}\right|-\frac{1}{|z| \Gamma\left(1-2 h_{k} / H\right)}-\frac{H^{2} K}{4 h_{k}^{2}|z|^{2}} \\
\text { for }-\frac{\pi}{H} \leqq \arg z \leqq-\frac{\pi}{H}+\frac{2 h_{k} \pi}{H}, \\
\left|g_{k}(z)\right| \leqq \frac{H}{2 h_{k}}\left|\exp \left(z \varepsilon^{\left(h_{k}-1\right) 2}\right)^{H / 2 h_{k}}\right|+\frac{1}{|z| \Gamma\left(1-2 h_{k} / H\right)}+\frac{H^{2} K}{4 h_{k}^{2}|z|^{2}} \\
\text { for }-\frac{\pi}{H}-\frac{h_{k} \pi}{2 H} \leqq \arg z \leqq-\frac{\pi}{H} \text { and for }-\frac{\pi}{H}+\frac{2 h_{k} \pi}{H} \leqq \arg z \leqq-\frac{\pi}{H}+\frac{5 h_{k} \pi}{2 H}, \\
\left|g_{k}(z)\right| \leqq \frac{1}{|z| \Gamma\left(1-2 h_{k} / H\right)}+\frac{H^{2} K}{4 h_{k}^{2}|z|} \\
\text { for }-\frac{\pi}{H}+\frac{5 h_{k} \pi}{2 H} \leqq \arg z \leqq 2 \pi-\frac{\pi}{H}-\frac{h_{k} \pi}{2 H}
\end{gathered}
$$

if $2 h_{k}<H$. If $2 h_{k}>H$, then we have

$$
\begin{aligned}
& \left|g_{k}(z)\right| \geqq\left\{\frac{H}{2\left(H-h_{k}\right)}\left|\exp \left(z \varepsilon^{\left(H+h_{k}-1\right)}\right)^{H /\left(2 H-2 h_{k}\right)}\right|+\frac{1}{|z| \Gamma\left(2 h_{k} / H-1\right)}+\frac{H^{2} K}{4\left(H-h_{k}\right)^{2}|z|^{2}}\right\}^{-1} \\
& \text { for } 2 \pi-\frac{\pi}{H} \leqq \arg z \leqq \frac{5 \pi}{2}-\frac{\pi}{H}-\frac{h_{k} \pi}{2 H} \text { and } \\
& \quad \text { for }-\frac{\pi}{2}-\frac{\pi}{H}-\frac{5 h_{k} \pi}{2 H} \leqq \arg z \leqq-\frac{\pi}{H}+\frac{2 h_{k} \pi}{H}, \\
& \left|g_{k}(z)\right| \geqq\left\{\frac{1}{|z| \Gamma\left(2 h_{k} / H-1\right)}+\frac{H^{2} K}{4\left(H-h_{k}\right)^{2}|z|^{2}}\right\}^{-1} \\
& \quad \text { for } \frac{\pi}{2}-\frac{\pi}{H}-\frac{h_{k} \pi}{2 H} \leqq \arg z \leqq-\frac{\pi}{2}-\frac{\pi}{H}+\frac{5 h_{k} \pi}{2 H}, \\
& \left|g_{k}(z)\right| \leqq\left\{\frac{H}{2\left(H-h_{k}\right)}\left|\exp \left(z \varepsilon^{\left(H+h_{k}-1\right)}\right)^{H /\left(2 I I-2 h_{k}\right)}\right|-\frac{1}{|z| \Gamma\left(2 h_{k} / H-1\right)}-\frac{H^{2} K}{4\left(H-h_{k}\right)^{2}|z|^{2}}\right\}^{-1} \\
& \text { for }-\frac{\pi}{H}-\frac{2 h_{k} \pi}{H} \leqq \arg z \leqq 2 \pi-\frac{\pi}{H} .
\end{aligned}
$$

Further if $2 h_{k}=H$, then we have

$$
\left|g_{k}(z)\right|=\left|\exp z \varepsilon^{\left(h_{k}-1\right) / 2}\right| \text {. }
$$

Thus, if $2 h_{k}<H$, then we have for sufficiently large $|z|$ 


$$
\begin{array}{ll}
\left|g_{k}(z)\right| \geqq \frac{1}{2} \cdot \exp \left(z \varepsilon^{\left(k k^{-1}\right) / 2}\right)^{H / 2 h_{k}} \mid & \text { for }-\frac{\pi}{H} \leqq \arg z \leqq-\frac{\pi}{H}+\frac{2 h_{k} \pi}{H}, \\
\left|g_{k}(z)\right| \leqq H & \text { for }-\frac{\pi}{H}+\frac{2 h_{k} \pi}{H} \leqq \arg z \leqq 2 \pi-\frac{\pi}{H} .
\end{array}
$$

(6) $\quad\left|g_{k}(z)\right| \leqq H$

If $2 h_{k}>H$, then we have for sufficiently large $|z|$

$$
\begin{aligned}
\left|g_{k}(z)\right| \geqq & \left\{H\left|\exp \left(z \varepsilon^{\left(H+h_{k}-1\right)}\right)^{H /\left(2 H-2 h_{k}\right)}\right|+\frac{1}{|z|}\right\}^{-1} \\
& \text { for } 2 \pi-\frac{\pi}{H} \leqq \arg z \leqq \frac{5 \pi}{2}-\frac{\pi}{H}-\frac{h_{k} \pi}{2 H} \text { and } \\
& \text { for }-\frac{\pi}{2}-\frac{\pi}{H}+\frac{5 h_{k} \pi}{2 H} \leqq \arg z \leqq-\frac{\pi}{H}+\frac{2 h_{k} \pi}{H},
\end{aligned}
$$

(8) $\quad\left|g_{k}(z)\right| \geqq|z| \quad$ for $\frac{\pi}{2}-\frac{\pi}{H}-\frac{h_{k} \pi}{2 H} \leqq \arg z \leqq-\frac{\pi}{2}-\frac{\pi}{H}+\frac{5 h_{k} \pi}{2 H}$,

(9) $\quad\left|g_{k}(z)\right| \leqq 1 \quad$ for $-\frac{\pi}{H}+\frac{2 h_{k} \pi}{H} \leqq \arg z \leqq 2 \pi-\frac{\pi}{H}$.

If $2 h_{k}=H$, then we have

(11) $\quad\left|g_{k}(z)\right| \leqq 1$

$$
\begin{aligned}
& \left|g_{k}(z)\right|=\left|\exp z \varepsilon^{\left(h_{k}-1\right) / 2}\right| \text { for }-\frac{\pi}{H} \leqq \arg z \leqq-\frac{\pi}{H}+\frac{2 h_{k} \pi}{H} \\
& \left|g_{k}(z)\right| \leqq 1 \quad \text { for }-\frac{\pi}{H}+\frac{2 h_{k} \pi}{H} \leqq \arg z \leqq 2 \pi-\frac{\pi}{H}
\end{aligned}
$$

Therefore from (3) and (5), for sufficiently large $M$, the set $\left\{z ;\left|\tilde{f}_{k}(z)\right|>M\right\}$ contains an unbounded region

$$
\tilde{G}_{k}(M) \equiv\left\{z ;\left|\cosh z^{H / 2} \exp \left(z \varepsilon^{(h k-1)}\right)^{H / 2 h_{k}}\right|>M^{2},-\frac{\pi}{H} \leqq \arg z \leqq-\frac{2 h_{k} \pi}{H}\right\}
$$

when $2 h_{k}<H$. Or from (3), (7) and (8), for sufficiently $M$, the set $\left\{z ;\left|\tilde{f}_{k}(z)\right|>M\right\}$ contains an unbounded region $\tilde{G}_{k}(M)$ which is a union of regions

$$
\begin{gathered}
\left\{z ;\left|\cosh z^{H / 2}\right|\left\{H\left|\exp \left(z \varepsilon^{\left(H+h_{k}-1\right)}\right)^{H /\left(2 H-2 h_{k}\right)}\right|+\frac{1}{|z|}\right\}^{-1}>M^{2},\right. \\
\left.2 \pi-\frac{\pi}{H} \leqq \arg z \leqq \frac{5 \pi}{2}-\frac{\pi}{H}-\frac{h_{k} \pi}{2 H} \text { and }-\frac{\pi}{2}-\frac{\pi}{H}+\frac{5 h_{k} \pi}{2 H} \leqq \arg z \leqq-\frac{\pi}{H}-\frac{2 h_{k} \pi}{H}\right\}
\end{gathered}
$$

and

$$
\left\{z ;\left|z \cosh z^{H / 2}\right|>M^{2}, \frac{\pi}{2}-\frac{\pi}{H}-\frac{h_{k} \pi}{2 H} \leqq \arg z \leqq-\frac{\pi}{2}-\frac{\pi}{H}+\frac{5 h_{k} \pi}{2 H}\right\}
$$

when $2 h_{k}>H$. Or from (3) and (10), for sufficiently large $M$, the set $\left\{z ;\left|\tilde{f}_{k}(z)\right|>M\right\}$ contains an unbounded region 


$$
\tilde{G}_{k}(M) \equiv\left\{z ;\left|\cosh z^{H / 2} \exp \left(z \varepsilon^{\left(h_{k}-1\right) / 2}\right)\right|>M^{2},-\frac{\pi}{H} \leqq \arg z \leqq-\frac{\pi}{H}+\frac{2 h_{k} \pi}{H}\right\}
$$

when $2 h_{k}=H$. Moreover we have

$$
\left|\tilde{f}_{k}(z)\right| \geqq\left|\cosh z^{H / 2}\right|
$$

in the set $\left\{z ;\left|\cosh z^{H / 2}\right|>M^{2},-\pi / H<\arg z<-\pi / H+2 h_{k} \pi / H\right\}$ which is contained in $\tilde{G}_{k}(M)$ for every $k$. Further from (4), (6), (9) and (11) we have

$$
\left|\tilde{f}_{k}(z)\right| \leqq H^{2} \quad \text { for }-\frac{\pi}{H}+\frac{2 h_{k} \pi}{H} \leqq \arg z \leqq 2 \pi-\frac{\pi}{H}
$$

for every $k$.

Now we define an asymptotic spot $\sigma_{k}$ over $w_{k}$ as follows. Let $G_{k}$ be the obtained from $\tilde{G}_{k}$ by the rotation $z \rightarrow z \varepsilon^{h_{1+\cdots}+h_{k-1}}$. Then for $z \in G_{k}\left(M^{2}\right)$ we have

$$
\left|\tilde{f}_{k}\left(z \varepsilon^{h_{1}+\cdots+h_{k-1}}\right)\right|>M^{2}
$$

and

$$
\left|\tilde{f}_{j}\left(z \varepsilon^{h_{1}+\cdots+h_{\jmath-1}}\right)\right|<H^{2} \quad(j=1, \cdots, k-1, k+1, \cdots, n),
$$

and hence for a sufficiently large $M$

$$
\begin{aligned}
& \left|f(z)-w_{k}\right| \\
\leqq & \left\{\sum_{j \neq k}\left|w_{j}-w_{k}\right|\left|\tilde{f}_{j}\left(z \varepsilon^{h_{1}+\cdots+h \jmath-1}\right)\right|+|A|\right\} /\left\{\left|\tilde{f}_{k}\left(z^{h^{1+\cdots+h+h-1}}\right)\right|-\sum_{j \neq k}\left|\tilde{f}_{j}\left(z \varepsilon^{h_{1}+\cdots+h_{\jmath-1}}\right)\right|\right\} \\
\leqq & \left\{\sum_{j \neq k}\left|w_{j}-w_{k}\right| H^{2}+|A|\right\} /\left(M^{2}-H^{3}\right) \\
\leqq & \frac{1}{M} .
\end{aligned}
$$

Therefore the set $\left\{z ;\left|f(z)-w_{k}\right|<1 / M\right\}$ contains the region $G_{k}\left(M^{2}\right)$. We then define $\sigma_{k}\left(\left|w-w_{k}\right|<1 / M\right)$ as the compoment of $\left\{z ;\left|f(z)-w_{k}\right|<1 / M\right\}$ containing $G_{k}\left(M^{2}\right)$. Further we see that all the spots $\sigma_{k}, k=1, \cdots, n$, are different each other. In fact, by (13) we have

$$
\begin{aligned}
\left|f(z)-w_{k}\right| & \geqq\left\{|A|-\sum_{j \neq k}\left|w_{j}-w_{k}\right|\left|\tilde{f}_{j}\left(z \varepsilon^{h_{1}+\cdots+h_{\jmath-1}}\right)\right|\right\} / \sum_{j=1}^{n}\left|\tilde{f}_{j}\left(z \varepsilon^{h_{1}+\cdots+h_{J-1}}\right)\right| \\
& \geqq\left\{|A|-\sum_{j \neq k}\left|w_{j}-w_{k}\right| H^{2}\right\} / n H^{2}
\end{aligned}
$$

on the half rays $\left\{z ; \arg z=-\pi / H+2\left(h_{1}+\cdots+h_{k-1}\right) \pi / H\right\}$ and $\left\{z ; \arg z=-\pi / H+2\left(h_{1}+\cdots\right.\right.$ $\left.\left.+h_{k}\right) \pi / H\right\}$ and if $A$ is a sufficiently large constant there exists a positive number $d$ such that $\left|f(z)-w_{k}\right|>d>0$.

We next show that $h\left(\sigma_{k}\right) \geqq h_{k}$. In $G_{k}\left(M^{2}\right)$ we have

$$
\frac{1}{M\left|f(z)-w_{k}\right|}
$$




$$
\begin{aligned}
& \geqq\left\{\left|\tilde{f}_{k}\left(z \varepsilon^{h_{1}+\cdots+h_{k-1}}\right)\right|-\sum_{j \neq k}\left|\tilde{f}_{j}\left(z \varepsilon^{h_{1}+\cdots+h_{\jmath-1}}\right)\right|\right\} / M\left(|A|+\sum_{j \neq k}\left|w_{j}-w_{k}\right| \mid \tilde{f}_{j}\left(z \varepsilon^{h_{1}+\cdots+h_{J-1}}\right)\right) \\
& \geqq\left\{\left|\tilde{f}_{k}\left(z \varepsilon^{h_{1}+\cdots+h_{k-1}}\right)\right|-n H^{2}\right\} / M\left(|A|+\sum_{j \neq k}\left|w_{j}-w_{k}\right| H^{2}\right) \\
& \geqq\left|\tilde{f}_{k}\left(z \varepsilon^{h_{1}+\cdots+h_{k-1}}\right)\right| / M^{2}
\end{aligned}
$$

for a sufficiently large $M$. By (12) and by Lemma, the function

$$
u_{\sigma_{k}\left(\left|w-w_{k}\right|<1 / M\right)}(z) \equiv \text { G.H.M. } \frac{1}{M\left|f(z)-w_{k}\right|}
$$

dominates at least $h_{k}$ mutually non-proportional minimal functions. Therefore $h\left(\sigma_{k},\left|w-w_{k}\right|<1 / M\right) \geqq h_{k}$ and hence $h\left(\sigma_{k}\right) \geqq h_{k}$.

The order $\rho$ of $f(z)$ is at most $H / 2$ since $f_{j}(z)$ is at most of order $H / 2$ and $g_{k}(z)$ is of order $H / 2 h_{k}$ or $H /\left(2 H-2 h_{k}\right)$. On the other hand, we get, by Theorem A, $\bar{H} \leqq 2 \rho$ for the grand total $\bar{H}$ of the harmonic indices of all the asymptotic spots of $f$. Consequently we have

$$
H=h_{1}+\cdots+h_{n} \leqq h\left(\sigma_{1}\right)+\cdots+h\left(\sigma_{n}\right) \leqq \bar{H} \leqq 2 \rho \leqq H,
$$

and hence

$$
\rho=\frac{H}{2}, h\left(\sigma_{1}\right)=h_{1}, \cdots, h\left(\sigma_{n}\right)=h_{n}, \text { and } \bar{H}=H .
$$

We thus have the desired result.

Finally as a direct consequence of Propositions 1 and 2, we have the following theorem:

Theorem. Let $w_{1}, \cdots, w_{n}$ denote $n(\geqq 1)$ given points on the extended plane and $h_{1}, \cdots, h_{n}$ denote $n$ given positive integers. Then there exists a meromorphic function $f(z)$ in $|z|<\infty$ which satisfies (I) the asymptotic spots of $f(z)$ with positive harmonic indices are $n$ in number, say $\sigma_{1}, \cdots, \sigma_{n}$, (II) $\sigma_{k}$ lies over $w_{k}$ and $h\left(\sigma_{k}\right)=h_{k}$, (III) $f(z)$ is of order $H / 2$, where $H=\sum_{k=1}^{n} h_{k}$.

The author wishes to express his thanks to Professor M. Ozawa who gave him valuable advices.

\section{REFERENCES}

[1] Cartwright, M. L., Integral functions. Cambridge (1962).

[2] Heins, M., On the Lindelöf principle. Ann. of Math. 61 (1955), 440-473.

[3] Heins, M., Aspmptotic spots of entire and meromorphic functions. Ann. of Math. 66 (1957), 430-439.

Department of Mathematics, Tokyo Institute of Technology. 